\title{
Markov Chain Monte Carlo Based on Deterministic Transformations
}

\author{
Somak Dutta* \\ Department of Statistics \\ University of Chicago \\ and \\ Sourabh Bhattacharya \\ Bayesian and Interdisciplinary Research Unit \\ Indian Statistical Institute
}

\begin{abstract}
In this article we propose a novel MCMC method based on deterministic transformations $T$ : $\mathcal{X} \times \mathcal{D} \rightarrow \mathcal{X}$ where $\mathcal{X}$ is the state-space and $\mathcal{D}$ is some set which may or may not be a subset of $\mathcal{X}$. We refer to our new methodology as Transformation-based Markov chain Monte Carlo (TMCMC). One of the remarkable advantages of our proposal is that even if the underlying target distribution is very high-dimensional, deterministic transformation of a one-dimensional random variable is sufficient to generate an appropriate Markov chain that is guaranteed to converge to the high-dimensional target distribution. Apart from clearly leading to massive computational savings, this idea of deterministically transforming a single random variable very generally leads to excellent acceptance rates, even though all the random variables associated with the high-dimensional target distribution are updated in a single block. Since it is well-known that joint updating of many random variables using Metropolis-Hastings ( $\mathrm{MH}$ ) algorithm generally leads to poor acceptance rates, TMCMC, in this regard, seems to provide a significant advance. We validate our proposal theoretically, establishing the convergence properties. Furthermore, we show that TMCMC can be very effectively adopted for simulating from doubly intractable distributions.

We show that TMCMC includes hybrid Monte Carlo (HMC) as a special case. We also contrast TMCMC with the generalized Gibbs and Metropolis methods of Liu and Yu (1999), Liu and Sabatti (2000) and Kou et al. (2005), pointing out that even though the latter also use transformations, their goal is to seek improvement of the standard Gibbs and Metropolis Hastings algorithms by adding a transformation-based step, while TMCMC is an altogether new and general methodology for simulating from intractable, particularly, high-dimensional distributions.

TMCMC is compared with MH using the well-known Challenger data, demonstrating the effectiveness of of the former in the case of highly correlated variables. Moreover, we apply our methodology to a challenging posterior simulation problem associated with the geostatistical model of Diggle
\end{abstract}

${ }^{*}$ Corresponding e-mail: sdutta@galton.uchicago.edu 
et al. (1998), updating 160 unknown parameters jointly, using a deterministic transformation of a one-dimensional random variable. Remarkable computational savings as well as good convergence properties and acceptance rates are the results.

Keywords: Geostatistics; High dimension; Inverse transformation; Jacobian; Metropolis-Hastings algorithm; Mixture proposal

\section{Introduction}

Markov chain Monte Carlo (MCMC) has revolutionized statistical, particularly, Bayesian computation. In the Bayesian paradigm, however complicated the posterior distribution may be, it is always possible, in principle, to obtain as many (dependent) samples from the posterior as desired, to make inferences about posterior characteristics. But in spite of the obvious success story enjoyed by the theoretical side of MCMC, satisfactory practical implementation of MCMC often encounters severe challenges, particularly in very high-dimensional problems. These challenges may arise in the form of the requirement of enormous computational effort, often requiring inversions of very high-dimensional matrices, implying the requirement of enormous computation time, even for a single iteration. Given that such high-dimensional problems typically converge extremely slowly to the target distribution triggered by complicated posterior dependence structures between the unknown parameters, astronomically large number of iterations (of the order of millions) are usually necessary. This, coupled with the computational expense of individual iterations, generally makes satisfactory implementation of MCMC, and hence, satisfactory Bayesian inference, infeasible. That this is the situation despite steady technological advancement, is somewhat disconcerting.

\subsection{Overview of the contributions of this paper}

In an attempt to overcome the problems mentioned above, in this paper we propose a novel methodology that can jointly update all the unknown parameters without compromising the acceptance rate, unlike in Metropolis-Hastings (MH) algorithm. In fact, we show that even though a very large number of parameters are to be updated, these can be updated by simple deterministic transformations of a single, one-dimensional random variable, the distribution of which can be chosen very flexibly. As can be already anticipated from this brief description, indeed, this yields an extremely fast simulation algorithm, thanks to the singleton random variable to be flexibly simulated, and the subsequent simple 
deterministic transformation, for example, additive transformation. It is also possible, maybe more efficient sometimes, to generate more than one, rather than a single, random variables, from a flexible multivariate (generally independent), but low-dimensional distribution. We refer to our new methodology as Transformation-based MCMC (TMCMC).

We show that by generating as many random variables as the number of parameters, instead of a single/few random variables, TMCMC can be reduced to a $\mathrm{MH}$ algorithm with a specialized proposal distribution. Another popular MCMC methodology, the hybrid Monte Carlo (HMC) method, which relies upon a specialized deterministic transformation, will be shown to be a special case of TMCMC.

We also provide a brief overview of the transformation-based generalized Gibbs and Metropolis methods of Liu and Yu (1999), Liu and Sabatti (2000) and Kou et al. (2005), and point out their differences with TMCMC, also arguing that TMCMC can be far more efficient at least in terms of computational gains.

Apart from illustrating TMCMC on the well-known Challenger data set, and demonstrating its superiority over existing MH methods, we successfully apply TMCMC with the mere simulation of a single random variable, to update 160 unknown parameters in every iteration, in the challenging geospatial problem of Diggle et al. (1998). The computational challenges involved with this and similar geospatial problems have motivated varieties of MCMC algorithms and deterministic approximations to the posterior in the literature (see, e. g. Rue (2009), Christensen (2006) and the references therein). With our TMCMC algorithm we have been able to perform $5.5 \times 10^{7}$ iterations (in a few days) and obtain reasonable convergence.

We also show how TMCMC can be adopted to significantly improve computational efficiency in doubly intractable problems, where the posterior, apart from being intractable, also involves the normalizing constant of the likelihood-the crucial point being that the normalizing constant, which depends upon unknown parameters, is also intractable.

The rest of this article is structured as follows. In Section 2 we introduce our new TMCMC method based on transformations. The univariate and the multivariate cases are considered separately in Sections 2.1 and 2.2 respectively. In Section 3 we study in details the role and efficiency of a singleton $\epsilon$ in updating high-dimensional Markov chains using TMCMC. Illustration of TMCMC with singleton $\epsilon$ using the Challenger data and comparison with a popular MCMC technique are provided in Section 4. Application of TMCMC with single $\epsilon$ to the 160-dimensional geospatial problem of Diggle et al. (1998) 
is detailed in Section 5. Section 6 shows how TMCMC may be applied to the bridge-exchange algorithm of Murray et al. (2006) in doubly intractable problems to speed-up computation. Finally, conclusions and overview of future work are provided in Section 7.

Further investigations and additional details are provided in the supplement Dutta and Bhattacharya (2013), whose sections, figures and tables have the prefix "S-" when referred to in this paper. The contents of the supplement are as follows. Section S-1 contains the proof of detailed balance for TMCMC, Section S-2 provides the general TMCMC algorithm for a one-dimensional proposal, while Section S3 contains details on convergence properties of additive TMCMC. In Section S-4 we provide a more structured version of the general TMCMC algorithm of Section S-2, proving detailed balance of this algorithm in Section S-5. Detailed investigation of acceptance rate of additive TMCMC and comparison with that of random walk $\mathrm{MH}(\mathrm{RWMH})$ is carried out in Section S-6. In Sections S-7 and S-8 respectively, comparisons of TMCMC with HMC and generalized Gibbs/Metropolis methods of Liu and Yu (1999), Liu and Sabatti (2000), and Kou et al. (2005) are provided. Examples of TMCMC for discrete state spaces are provided in Section S-9.

\section{MCMC algorithms based on transformations on the state-space}

In this section we propose and study the TMCMC algorithms. First, we construct it for state-spaces of dimension one. This case is not of much interest because the state space is similar to the real line and numerical integration is quite efficient in this scenario. Nevertheless, construction of the TMCMC algorithm for one dimensional problems helps to generalize it to higher dimensions and points out its connections (similarities in one-dimension and dissimilarities in higher dimensions) with the MH algorithm. In Sections 2.2 and 3 the TMCMC algorithm is generalized to higher dimensional state-spaces, the latter section considering the utility of single $\epsilon$ in high dimensions.

\subsection{Univariate case}

Before providing the formal theory we first provide an informal discussion of our ideas with a simple example involving the additive transformation. 


\subsubsection{Informal discussion}

In order to obtain a valid algorithm based on transformations, we need to design appropriate "move types" so that detailed balance and irreducibility hold. Given that we are in the current state $x$, we can propose the "forward move" $x^{\prime}=x+\epsilon$; here $\epsilon>0$ is a simulation from some arbitrary density of the form $g(\epsilon) I_{(0, \infty)}(\epsilon)$. To move back to $x$ from $x^{\prime}$, we need to apply the "backward transformation" $x^{\prime}-\epsilon$. In general, given $\epsilon$ and the current state $x$, we shall denote the forward transformation by $T(x, \epsilon)$, and the backward transformation by $T^{b}(x, \epsilon)$.

The forward and the backward transformations need to be 1-to-1. In other words, for any fixed $\epsilon$, given $x^{\prime}$ the backward transformation must be such that $x$ can be retrieved uniquely. Since this must hold good for every $x$ in the state space, the transformation must be onto as well. Similarly, for any fixed $\epsilon$, there must exist $x$ such that the forward transformation leads to arbitrarily chosen $x^{\prime}$ in the state space uniquely, implying that this transformation is also 1-to-1 and onto. If, given $\epsilon$ and $x^{\prime}$, say, more than one solution exist, then return to the current value $x$ can not be ensured, and this makes detailed balance, a requirement for stationarity of the underlying Markov chain, hard to satisfy.

The detailed balance requirement also demands that, given $x$, the regions covered by the forward and the backward transformations are disjoint. For example, in our additive transformation case, the forward transformation always takes $x$ to some unique $x^{\prime}$, where $x^{\prime}>x$. To return from $x^{\prime}$ to $x$, it is imperative that the backward transformation decreases the value of $x^{\prime}$ to give back $x$. Thus, if the forward transformation always increases the current value $x$, the backward transformation must always decrease $x$. In other words, the regions covered by the two transformations are disjoint. Since $x$ is led to $x^{\prime}$ by the forward transformation and $x^{\prime}$ is taken back to $x$ by the backward transformation, we must have $T\left(T^{b}(x, \epsilon), \epsilon\right)=x$. Also, the sequence of forward and backward transformations can be changed to achieve the same effect, that is, we must also have $T^{b}(T(x, \epsilon), \epsilon)=x$. In the above discussion we indicated the use the same $\epsilon$ for updating $x$ to $x^{\prime}$ and for moving back from $x^{\prime}$ to $x$. An important advantage associated with this strategy is that whatever the choice of the density $g(\epsilon) I_{(0, \infty)}(\epsilon)$, it will cancel in the acceptance ratio of our TMCMC algorithm, resulting in a welcome simplification.

Thanks to bijection each of the forward and the backward transformations will be equipped with their respective inverses. In general, we denote by $T(x, \epsilon)$ and $T^{b}(x, \epsilon)$ the forward and the backward transformations, and by $T^{-1}(x, \epsilon)$ and $T^{b^{-1}}(x, \epsilon)$ their respective inverses. Note that for fixed $\epsilon$, $T^{-1}(x, \epsilon)=T^{b}(x, \epsilon)$, and $T^{b^{-1}}(x, \epsilon)=T(x, \epsilon)$, but the general inverses must be defined by eliminating 
$\epsilon$. For instance, substituting $\epsilon=x^{\prime}-x$ for the forward transformation yields $T(x, \epsilon)=T\left(x, x^{\prime}-x\right)=x+$ $\left(x^{\prime}-x\right)=x^{\prime}$. Defining $T^{-1}\left(x, x^{\prime}\right)=x^{\prime}-x$, it then follows that $T\left(x, T^{-1}\left(x, x^{\prime}\right)\right)=x^{\prime}=T^{-1}\left(x, T\left(x, x^{\prime}\right)\right)$, showing that $T^{-1}$ is the inverse of $T$ in the above sense. Similarly, $T^{b^{-1}}$ can also be defined.

\subsubsection{Formal set-up}

Suppose $T: \mathcal{X} \times \mathcal{D} \rightarrow \mathcal{X}$ for some $\mathcal{D}$ (possibly a subset of $\mathcal{X}$ ) is a totally differentiable transformation such that

1. for every fixed $\epsilon \notin \mathcal{N}_{1}$, the transform $x \longmapsto T(x, \epsilon)$ is bijective and differentiable and that the inverse is also differentiable.

2. for every fixed $x \notin \mathcal{N}_{2}$, the transform $\epsilon \longmapsto T(x, \epsilon)$ is injective.

where $\mathcal{N}_{1}$ and $\mathcal{N}_{2}$ are $\pi$-negligible sets. Further suppose that the Jacobian

$$
J(x, \epsilon)=\left|\frac{\partial(T(x, \epsilon), \epsilon)}{\partial(x, \epsilon)}\right|
$$

is non-zero almost everywhere.

Suppose there is a subset $\mathcal{Y}$ of $\mathcal{D}$ such that $\forall x \notin \mathcal{N}_{2}$ the sets $T(x, \mathcal{Y})$ and $T^{b}(x, \mathcal{Y})$ are disjoint, where $T^{b}(x, \epsilon)$ is the backward transformation defined by:

$$
T\left(T^{b}(x, \epsilon), \epsilon\right)=T^{b}(T(x, \epsilon), \epsilon)=x
$$

\section{Example: Transformations on One dimensional state-space}

1. (additive transformation) Suppose $\mathcal{X}=\mathcal{D}=\mathbb{R}$ and $T(x, \epsilon)=x+\epsilon$. Let $T^{b}(x, \epsilon)=x-\epsilon$. This transformation is basically the random walk if $\epsilon$ is a random quantity. Notice that if we may choose $\mathcal{Y}=(0, \infty)$, then $T(x, \mathcal{Y})=(x, \infty), T^{b}(x, \mathcal{Y})=(-\infty, x)$ and we can characterize the transformation as a forward move or a backward move according as $\epsilon \in$ or $\notin \mathcal{Y}$. Notice that here $\mathcal{N}$ is the empty set and for all $\epsilon \in \mathcal{D}$ the map $x \longmapsto T(x, \epsilon)$ is a bijection.

2. (log-additive transformation) Suppose $\mathcal{X}=\mathcal{D}=(0, \infty)$ and $T(x, \epsilon)=x \epsilon$. For all $x \in \mathcal{X}$, $T^{b}(x, \epsilon)=x / \epsilon ; \mathcal{Y}=(0,1)$.

3. (multiplicative transformation) Let $\mathcal{X}=\mathbb{R}=\mathcal{D}, T(x, \epsilon)=x \epsilon$. Then $\mathcal{N}_{1}=\mathcal{N}_{2}=\{0\}$, for all $\epsilon \neq 0, T^{b}(x, \epsilon)=x / \epsilon ; \mathcal{Y}=(-1,1)-\{0\}$. 
Suppose further that $g$ is a density on $\mathcal{Y}$ and that $0<p<1$. Then the MCMC algorithm based on transformation is given in Algorithm 2.1

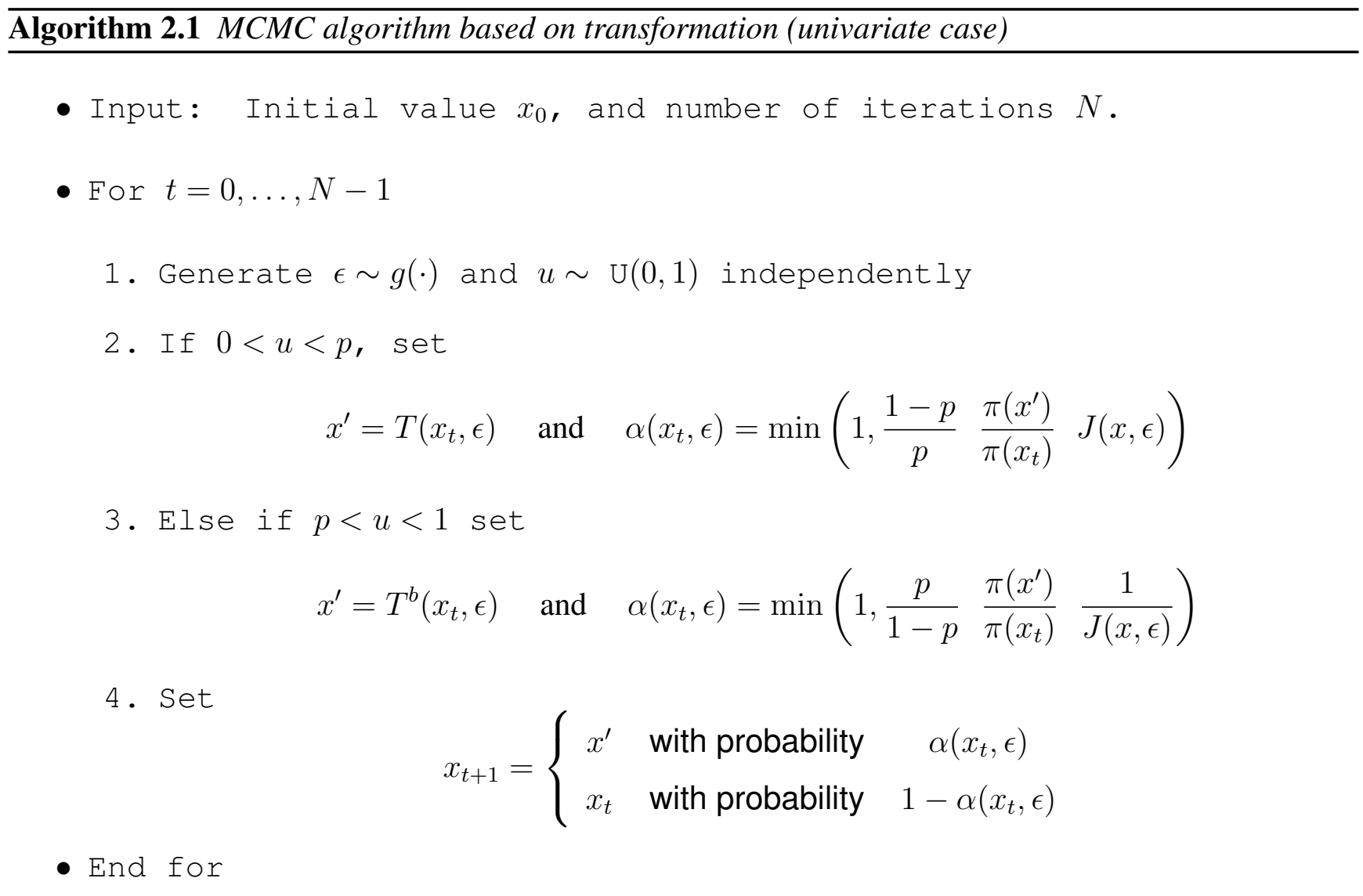

Notably, the acceptance probability is independent of the distribution $g(\cdot)$, even if it is not symmetric.

The algorithm can be shown to be a special case of $\mathrm{MH}$ algorithm with the mixture proposal density:

$$
\begin{array}{rl}
q(x \rightarrow z)=p & g\left(T^{-1}(x, z)\right)\left|\frac{\partial T^{-1}(x, z)}{\partial z}\right| \mathbb{I}(z \in T(x, \mathcal{Y})) \\
+(1-p) g\left(T^{b^{-1}}(x, z)\right)\left|\frac{\partial T^{b^{-1}}(x, z)}{\partial z}\right| \mathbb{I}\left(z \in T^{b}(x, \mathcal{Y})\right)
\end{array}
$$

where the inverses are defined by

1. $T\left(x, T^{-1}(x, z)\right)=z=T^{-1}(x, T(x, z)), \forall z \in T(x, \mathcal{Y})$

2. $T^{b}\left(x, T^{b^{-1}}(x, z)\right)=z=T^{b^{-1}}\left(x, T^{b}(x, z)\right), \forall z \in T^{b}(x, \mathcal{Y})$ 
In Section S-1 we show that detailed balance holds for the above algorithm. This ensures that our TMCMC methodology has $\pi$ as the stationary distribution. Although in this univariate case TMCMC is an $\mathrm{MH}$ algorithm with the specialized mixture density (2.1) as the proposal mechanism, this proposal distribution becomes singular in general in higher dimensions.

We remark that TMCMC maybe particularly useful for improving the mixing properties of the Markov chain. For instance, if there are distinct modes in several disjoint regions of state space, then standard MH algorithms tend to get trapped in some modal regions, particularly if the proposal distribution has small variance. Higher variance, on the other hand, may lead to poor acceptance rates in standard MH algorithms. Gibbs sampling is perhaps more prone to mixing problems due to the lack of tuning facilities. For multimodal target distributions, mixture proposal densities are often recommended. For instance, Guan and Krone (2007) theoretically prove that a mixture of two proposal densities results in a "rapidly mixing" Markov chain when the target distribution is multimodal. Our proposal, which we have shown to be a mixture density in the one-dimensional case, seems to be appropriate from this perspective. Indeed, in keeping with this discussion, Dutta (2012), apart from showing that the multiplicative transformation is geometrically ergodic even in situations where the standard proposals fail to be so, demonstrated that it is very effective for bimodal distributions. These arguments demonstrate that a real advantage of TMCMC (also of other transformation-based methods as in Liu (2001)) comes forth when the transformations associated with our method identify a subspace moving within which allows to explore regions that are otherwise separated by valleys in the probability function. Efficient choice of transformations of course depends upon the target distribution.

In higher dimensions our proposal does not admit a mixture form but since the principles are similar, it is not unreasonable to expect good convergence properties of TMCMC in the cases of high-dimensional and/or multimodal target densities. In the multidimensional case, which makes use of multivariate transformations (which we introduce next), reasonable acceptance rates can also be ensured, in spite of the high dimensionality. This we show in Section S-6, and illustrate with the Challenger data problem and particularly with the geostatistical problem. Moreover, the multivariate transformation method brings out other significant advantages of our method, for instance, computational speed and the ability to overcome mixing problems caused by highly correlated variables. 


\subsection{Multivariate case}

Suppose now that $\mathcal{X}$ is a $k$-dimensional space of the form $\mathcal{X}=\prod_{i=1}^{k} \mathcal{X}_{i}$ so that $T=\left(T_{1}, \ldots, T_{k}\right)$ where each $T_{i}: \mathcal{X}_{i} \times \mathcal{D} \rightarrow \mathcal{X}_{i}$, for some set $\mathcal{D}$, are transformations as in Section 2.1. Let $\mathbf{z}=\left(z_{1}, \ldots, z_{k}\right)$ be a vector of indicator variables, where, for $i=1, \ldots, k, z_{i}=1$ and $z_{i}=-1$ indicate, respectively, application of forward transformation and backward transformation to $x_{i}$. Given any such indicator vector $\mathbf{z}$, let us define $T_{\mathbf{z}}=\left(g_{1}, g_{2}, \ldots, g_{k}\right)$ where

$$
g_{i}=\left\{\begin{array}{ccc}
T_{i}^{b} & \text { if } & z_{i}=-1 \\
T_{i} & \text { if } & z_{i}=1
\end{array}\right.
$$

Corresponding to any given $\mathbf{z}$, we also define the following 'conjugate' vector $\mathbf{z}^{c}=\left(z_{1}^{c}, z_{2}^{c}, \ldots, z_{k}^{c}\right)$, where

$$
z_{i}^{c}=\left\{\begin{array}{ccc}
1 & \text { if } & z_{i}=-1 \\
-1 & \text { if } & z_{i}=1
\end{array}\right.
$$

With this definition of $\mathbf{z}^{c}, T_{\mathbf{z}^{c}}$ can be interpreted as the conjugate of $T_{\mathbf{z}}$.

Since $2^{k}$ values of $\mathbf{z}$ are possible, it is clear that $T$, via $\mathbf{z}$, induces $2^{k}$ many types of 'moves' of the forms $\left\{T_{\mathbf{z}_{i}} ; i=1, \ldots, 2^{k}\right\}$ on the state-space. Suppose now that there is a subset $\mathcal{Y}$ of $\mathcal{D}$ such that the sets $T_{\mathbf{z}_{i}}(\mathbf{x}, \mathcal{Y})$ and $T_{\mathbf{z}_{j}}(\mathbf{x}, \mathcal{Y})$ are disjoint for every $\mathbf{z}_{i} \neq \mathbf{z}_{j}$.

\section{Examples: Transformations on higher dimensional state-space}

1. (Additive transformation) Suppose $\mathcal{X}=\mathcal{D}=\mathbb{R}^{2}$. With two positive scale parameters $a_{1}$ and $a_{2}$, we can then consider the following additive transformation: $T_{(1,1)}(\mathbf{x}, \boldsymbol{\epsilon})=\left(x_{1}+a_{1} \epsilon_{1}, x_{2}+a_{2} \epsilon_{2}\right)$, $T_{(-1,1)}(\mathbf{x}, \boldsymbol{\epsilon})=\left(x_{1}-a_{1} \epsilon_{1}, x_{2}+a_{2} \epsilon_{2}\right), T_{(1,-1)}(\mathbf{x}, \boldsymbol{\epsilon})=\left(x_{1}+a_{1} \epsilon_{1}, x_{2}-a_{2} \epsilon_{2}\right)$ and $T_{(-1,-1)}(\mathbf{x}, \boldsymbol{\epsilon})=$ $\left(x_{1}-a_{1} \epsilon_{1}, x_{2}-a_{2} \epsilon_{2}\right)$. We may choose $\mathcal{Y}=(0, \infty) \times(0, \infty)$.

2. (Multiplicative transformation) Suppose $\mathcal{X}=\mathcal{D}=\mathbb{R} \times(0, \infty)$. Then we may consider the following multiplicative transformation: $T_{(1,1)}(\mathbf{x}, \boldsymbol{\epsilon})=\left(x_{1} \epsilon_{1}, x_{2} \epsilon_{2}\right), T_{(-1,1)}(\mathbf{x}, \boldsymbol{\epsilon})=\left(x_{1} / \epsilon_{1}, x_{2} \epsilon_{2}\right)$, $T_{(1,-1)}(\mathbf{x}, \boldsymbol{\epsilon})=\left(x_{1} \epsilon_{1}, x_{2} / \epsilon_{2}\right)$ and $T_{(-1,-1)}(\mathbf{x}, \boldsymbol{\epsilon})=\left(x_{1} / \epsilon_{1}, x_{2} / \epsilon_{2}\right)$. We may let $\mathcal{Y}=\{(-1,1)-\{0\}\} \times$ $(0,1)$.

3. (Additive-multiplicative transformation) Suppose $\mathcal{X}=\mathcal{D}=\mathbb{R} \times(0, \infty)$. It is possible to combine additive and multiplicative transformations in the following manner: $T_{(1,1)}(\mathbf{x}, \boldsymbol{\epsilon})=\left(x_{1}+\epsilon_{1}, x_{2} \epsilon_{2}\right)$, $T_{(-1,1)}(\mathbf{x}, \boldsymbol{\epsilon})=\left(x_{1}-\epsilon_{1}, x_{2} \epsilon_{2}\right), T_{(1,-1)}(\mathbf{x}, \boldsymbol{\epsilon})=\left(x_{1}+\epsilon_{1}, x_{2} / \epsilon_{2}\right)$ and $T_{(-1,-1)}(\mathbf{x}, \boldsymbol{\epsilon})=\left(x_{1}-\epsilon_{1}, x_{2} / \epsilon_{2}\right)$. We may let $\mathcal{Y}=(0, \infty) \times(0,1)$. 
The above examples can of course be generalized to arbitrary dimensions. Also, it is clear that it is possible to construct valid transformations in high-dimensional spaces using combinations of valid transformations on one-dimensional spaces.

Now suppose that $g$ is a density on $\mathcal{Y}$, and, for $i=1, \ldots, 2^{k}$, let $P_{i}=P\left(T_{\mathbf{z}_{i}}\right)$ be the probability of the move-type $T_{\mathbf{z}_{i}}$. We assume that for each $i, P_{i}>0$ and $\sum_{i=1}^{2^{k}} P_{i}=1$. Note that this requires us to specify the $2^{k}$-dimensional probability vector, which seems to be a daunting task for large $k$. However, in Section 3.1 we show that this difficulty can be overcome by considering a product form of the movetype probabilities induced by a mechanism of simulating $\mathbf{z}$, which facilitates the choice of appropriate move-types from the very large set of available move-types. This mechanism is also highly efficient computationally.

The MCMC algorithm based on transformations is given in Algorithm 2.2.

\section{Algorithm 2.2 MCMC algorithm based on transformation (multivariate case)}

- Input: Initial value $\mathbf{x}^{(0)}$, and number of iterations $N$.

- For $t=0, \ldots, N-1$

1. Generate $\boldsymbol{\epsilon} \sim g(\cdot)$ and an index $i \sim \mathcal{M}\left(1 ; P_{1}, \ldots, P_{2^{k}}\right)$ independently. Actually, simulation from the multinomial distribution is not necessary; see Section 3.1 for an efficient and computationally inexpensive method of generating the index even when the number of move-types far exceeds $2^{k}$.

2 .

$$
\mathbf{x}^{\prime}=T_{\mathbf{z}_{i}}\left(\mathbf{x}^{(t)}, \boldsymbol{\epsilon}\right) \quad \text { and } \quad \alpha\left(\mathbf{x}^{(t)}, \boldsymbol{\epsilon}\right)=\min \left(1, \frac{P\left(T_{\mathbf{z}_{i}^{c}}\right)}{P\left(T_{\mathbf{z}_{i}}\right)} \frac{\pi\left(\mathbf{x}^{\prime}\right)}{\pi\left(\mathbf{x}^{(t)}\right)}\left|\frac{\partial\left(T_{\mathbf{z}_{i}}\left(\mathbf{x}^{(t)}, \boldsymbol{\epsilon}\right), \boldsymbol{\epsilon}\right)}{\partial\left(\mathbf{x}^{(t)}, \boldsymbol{\epsilon}\right)}\right|\right)
$$

3. Set

$$
\mathbf{x}^{(t+1)}=\left\{\begin{array}{ccc}
\mathbf{x}^{\prime} & \text { with probability } & \alpha\left(\mathbf{x}^{(t)}, \boldsymbol{\epsilon}\right) \\
\mathbf{x}^{(t)} & \text { with probability } & 1-\alpha\left(\mathbf{x}^{(t)}, \boldsymbol{\epsilon}\right)
\end{array}\right.
$$

- End for 
In light of the above algorithm, it can be seen that for each of the transformations in the above examples, a mixture proposal of the form (2.1) is induced. It will, however, be pointed out in Section 3 that a singleton $\epsilon$ suffices for updating multiple random variables simultaneously, which would imply singularity of the underlying proposal distribution. Notice that for arbitrary dimensions the additive transformation reduces to the RWMH.

Algorithm 2.2 indicates that updating highly correlated variables can be done naturally with TMCMC: for instance, in Example 1 of this section one may select $T_{(1,1)}(\mathbf{x}, \boldsymbol{\epsilon})$ and $T_{(-1,-1)}(\mathbf{x}, \boldsymbol{\epsilon})$ with high probabilities if $x_{1}$ and $x_{2}$ are highly positively correlated and $T_{(-1,1)}(\mathbf{x}, \boldsymbol{\epsilon})$ and $T_{(1,-1)}(\mathbf{x}, \boldsymbol{\epsilon})$ may be selected with high probabilities if $x_{1}$ and $x_{2}$ are highly negatively correlated.

\section{Validity and usefulness of singleton $\epsilon$ in implementing TMCMC in high dimensions}

Crucially, a singleton $\epsilon$ suffices to ensure the validity of our algorithm, even though many variables are to be updated. This indicates a very significant computational advantage over all other MCMC-based methods: for instance, complicated simulation of hundreds of thousands of variables may be needed for any MCMC-based method, while, for the same problem, a single simulation of our methodology will do. Indeed, in Section 5 we update 160 variables using a single $\epsilon$ in the geostatistical problem of Diggle et al. (1998). This singleton $\epsilon$ also ensures that a mixture MH proposal density corresponding to our TMCMC method does not exist. The last fact shows that TMCMC can not be a special case of the MH algorithm. On the other hand, assuming that instead of singleton $\epsilon$, there is an $\epsilon_{i}$ associated with each of the variables $x_{i} ; i=1, \ldots, k$, then again TMCMC boils down to the $\mathrm{MH}$ algorithm, and, as in the univariate case, here also our transformations would induce a mixture proposal distribution for the algorithm, consisting of $2^{k}$ mixture components each corresponding to a multivariate transformation.

Using singleton $\epsilon$, for transformations other than the additive transformation, it is necessary to incorporate extra move types having positive probability which change one variable using forward or backward transformation, keeping the other variables fixed at their current values. Consider for instance, Example 3 of Section 2.2. The example indicates that, with a singleton $\epsilon$, it is only possible to move from $\left(x_{1}, x_{2}\right)$ to either of the following states: $\left(x_{1}+\epsilon, x_{2} \epsilon\right),\left(x_{1}-\epsilon, x_{2} \epsilon\right),\left(x_{1}+\epsilon, x_{2} / \epsilon\right)$ and $\left(x_{1}-\epsilon, x_{2} / \epsilon\right)$ with 
positive probabilities. In addition, we could specify that the states $\left(x_{1}, x_{2} \epsilon\right),\left(x_{1}, x_{2} / \epsilon\right),\left(x_{1}+\epsilon, x_{2}\right)$ and $\left(x_{1}-\epsilon, x_{2}\right)$ also have positive probabilities to be visited from $\left(x_{1}, x_{2}\right)$ in one step. We will need to specify the visiting probabilities $P_{i}>0 ; i=1, \ldots, 8$ such that $\sum_{i=1}^{8} P_{i}=1$. A general method of specifying the move-type probabilities, which also preserves computational efficiency, is discussed in Section 3.1. Inclusion of the extra move types ensures irreducibility and aperiodicity (the definitions are provided in Section S-3) of the Markov chain. It is easy to see that even for higher dimensions irreducibility and aperiodicity can be enforced by bringing in move types of similar forms that updates one variable keeping the remaining variables fixed. One only needs to bear in mind that the move types must be included in pairs, that is, a move type that updates only the $i$-th co-ordinate $x_{i}$ using forward transformation and the conjugate move type that updates only $x_{i}$ using the backward transformation both must have positive probability of selection.

With single $\epsilon$ and the addition of the extra move types Algorithm 2.2 requires only slight modification. As in Section 2.2 let $\mathbf{z}=\left(z_{1}, \ldots, z_{k}\right)$ be the vector of indicator variables, but now, in addition to the values 1 and -1 as before, $z_{i}$ can take the value 0 as well, indicating no change to $x_{i}$. The generalized definition of $z_{i}$ can be expressed as follows:

$$
z_{i}=\left\{\begin{array}{ccc}
1 & \text { indicates forward transformation to } & x_{i} \\
0 & \text { indicates no change to } & x_{i} \\
-1 & \text { indicates negative transformation to } & x_{i}
\end{array}\right.
$$

Given any such indicator vector $\mathbf{z}$, we define as before $T_{\mathbf{z}}=\left(g_{1}, g_{2}, \ldots, g_{k}\right)$ where now we extend the definition of $g_{i}$ to the following:

$$
g_{i}=\left\{\begin{array}{ccc}
T_{i}^{b} & \text { if } & z_{i}=-1 \\
x_{i} & \text { if } & z_{i}=0 \\
T_{i} & \text { if } & z_{i}=1
\end{array}\right.
$$

We also need to extend the definition of the conjugate vector: given $\mathbf{z}$, we define the conjugate vector $\mathbf{z}^{c}=\left(z_{1}^{c}, z_{2}^{c}, \ldots, z_{k}^{c}\right)$, where

$$
z_{i}^{c}=\left\{\begin{array}{ccc}
1 & \text { if } & z_{i}=-1 \\
0 & \text { if } & z_{i}=0 \\
-1 & \text { if } & z_{i}=1
\end{array}\right.
$$

In this definition of $z_{i}, 3^{k}$ values of $\mathbf{z}$ are possible, so that we now have $3^{k}$ possible move-types the forms $\left\{T_{\mathbf{z}_{i}} ; i=1, \ldots, 3^{k}\right\}$ on the state-space. Now note that the move type induced by $\mathbf{z}=(0,0, \ldots, 0)$ does 
not propose any change to the current state $\mathrm{x}$. Hence, we discard this move, and consider the remaining $3^{k}-1$ move-types for our TMCMC methodology. Now suppose that $g$ is a density on $\mathcal{Y}$, and, for $i=1, \ldots, 3^{k}-1$, let $P_{i}=P\left(\mathbf{z}_{i}\right)$ be the probability of the move-type $T_{\mathbf{z}_{i}}$. We assume that for each $i$, $P_{i}>0$ and $\sum_{i=1}^{3^{k}-1} P_{i}=1$.

With these minor modifications Algorithm (2.2) goes through with $\epsilon$ replaced by the singleton $\epsilon$. For the sake of completeness, we present our general TMCMC algorithm based on a single $\epsilon$ in Section S-2 (Algorithm S-2.1).

This strategy works for all transformations, including the examples in Section 2.2 where we now assume equality of all the components of $\epsilon$. Only additional move types are involved for transformations in general. However, we prove in Section S-3 that the additive transformation does not require the additional move types. Also taking account of the inherent simplicity of this transformation, the additive transformation is our automatic choice for the applications reported in this paper.

\subsection{Flexible and computationally efficient specification of the move-type proba- bilities}

An apparent drawback of Algorithms 2.2 and S-2.1 is the difficulty of specifying the move-type probabilities $p(\mathbf{z})$ for all possible values of $\mathbf{z}$. For large dimension $k$, manual specification of such highdimensional probability vector is clearly infeasible. Moreover, step 1 of Algorithms 2.2 and S-2.1 refers to simulation from a multinomial distribution involving the very high-dimensional move-type probability vector. But simulation from such a high-dimensional multinomial distribution can be computationally burdensome in the extreme if traditional methods of multinomial simulation are used, even if specification of the move-type probability vector is at all possible. In this section we show how both these problems can be avoided. The key idea is to note that the move-type probabilities of $T_{\mathbf{z}}$ can be induced by assigning probabilities to all possible values of $\mathbf{z}$; a simple, but useful way is to assign positive probabilities to $\{-1,0,1\}$, the possible values of each component $z_{i}$ of $z$. The latter induces a probability distribution on the set of available move-types $T_{\mathbf{z}}$, and hence on the high-dimensional multinomial distribution. Simulation of $\mathbf{z}$ by drawing $z_{i}$ independently for $i=1, \ldots, k$ yields the move-type $T_{\mathbf{z}}$, thus obviating the requirement of simulation from the high-dimensional multinomial distribution using traditional methods. In this mechanism specification of only the probabilities $\operatorname{Pr}\left(Z_{i}=1\right)$ and $\operatorname{Pr}\left(Z_{i}=-1\right)$ for $i=1 \ldots, k$, are required, which is manageable. Details follow. 
Consider a $k(\geq 1)$-dimensional target distribution, with associated random variables $\mathbf{x}=\left(x_{1}, \ldots, x_{k}\right)$. Then, we can implement the following simple rule. Given $\mathbf{x}$, let the forward and the backward transformations be applied to $x_{i}$ with probabilities $p_{i}$ and $q_{i}$, respectively. With probability $1-p_{i}-q_{i}, x_{i}$ remains unchanged. We now define $\mathbf{z}$ to be a random vector such that the random variable $z_{i}$ takes values $-1,0,1$, with probabilities $q_{i}, 1-p_{i}-q_{i}, p_{i}$, respectively. The values $-1,0,1$ correspond, as before, to backward transformation, no change, and forward transformation, respectively.

This rule, which is to be applied to each of $i=1, \ldots, k$ coordinates, includes all possible move types, including the one where none of the $x_{i}$ is updated, that is, $\mathbf{x}$ is taken to $\mathbf{x}$. Since the move-type $\mathbf{x} \mapsto \mathbf{x}$ is redundant, this is to be rejected whenever it appears. In other words, we would keep simulating the discrete random vector $\mathbf{z}=\left(z_{1}, \ldots, z_{k}\right)$ until at least one $z_{i} \neq 0$, and would then select the corresponding move type. For any dimension, this is a particularly simple and computationally efficient exercise, since the rejection region is a singleton, and has very small probability (particularly in high dimensions) if either of $p_{i}$ and $q_{i}$ is high for at least one $i$.

Since now we induce the probability distribution of $T_{\mathbf{z}}$ through $\mathbf{z}$, we denote $P\left(T_{\mathbf{z}}\right)$ by $P(\mathbf{z})$. The above method implies that the probability of a move-type, given $\mathbf{z}$, is of the form

$$
P(\mathbf{z})=C \prod_{\left\{i_{1}: z_{i_{1}}=1\right\}} p_{i_{1}} \prod_{\left\{i_{2}: z_{i_{2}}=-1\right\}} q_{i_{2}} \prod_{\left\{i_{3}: z_{i_{3}}=0\right\}}\left(1-p_{i_{3}}-q_{i_{3}}\right),
$$

and $C$ is the normalizing constant, which arose due to rejection of the move type $\mathbf{x} \mapsto \mathbf{x}$. This normalizing constant cancels in the acceptance ratio, and so it is not required to calculate it explicitly, another instance of preservation of computational efficiency. Note that the probability of the conjugate movetype is

$$
\begin{aligned}
P\left(\mathbf{z}^{c}\right) & =C \prod_{\left\{i_{1}: z_{i_{1}}^{c}=1\right\}} p_{i_{1}} \prod_{\left\{i_{2}: z_{i_{2}}^{c}=-1\right\}} q_{i_{2}} \prod_{\left\{i_{3}: z_{i_{3}}^{c}=0\right\}}\left(1-p_{i_{3}}-q_{i_{3}}\right) \\
& =C \prod_{\left\{i_{1}: z_{i_{1}}=-1\right\}} p_{i_{1}} \prod_{\left\{i_{2}: z_{i_{2}}=1\right\}} q_{i_{2}} \prod_{\left\{i_{3}: z_{i_{3}}=0\right\}}\left(1-p_{i_{3}}-q_{i_{3}}\right),
\end{aligned}
$$

so that the factor $\prod_{\left\{i_{3}: z_{i_{3}}=0\right\}}\left(1-p_{i_{3}}-q_{i_{3}}\right)$ cancels in the acceptance ratio, further simplifying computation. Algorithm 3.1 gives the simplified TMCMC algorithm based on a singleton $\epsilon$.

Algorithm 3.1 Simplified TMCMC algorithm based on a single $\epsilon$.

- Input: Initial value $\mathbf{x}^{(0)}$, and number of iterations $N$. 
- For $t=0, \ldots, N-1$

1. Generate $\epsilon \sim g(\cdot)$ and simulate $\mathbf{z}$ by generating $z_{i} \sim \mathcal{M}\left(1 ; p_{i}, q_{i}, 1-p_{i}-\right.$ $q_{i}$ ) independently for $i=1, \ldots, k$.

2 .

$$
\mathbf{x}^{\prime}=T_{\mathbf{z}}\left(\mathbf{x}^{(t)}, \epsilon\right) \quad \text { and } \quad \alpha\left(\mathbf{x}^{(t)}, \epsilon\right)=\min \left(1, \frac{P\left(\mathbf{z}^{c}\right)}{P(\mathbf{z})} \frac{\pi\left(\mathbf{x}^{\prime}\right)}{\pi\left(\mathbf{x}^{(t)}\right)}\left|\frac{\partial\left(T_{\mathbf{z}}\left(\mathbf{x}^{(t)}, \epsilon\right), \epsilon\right)}{\partial\left(\mathbf{x}^{(t)}, \epsilon\right)}\right|\right),
$$

where

$$
\frac{P\left(\mathbf{z}^{c}\right)}{P(\mathbf{z})}=\prod_{\left\{i_{1}: z_{i_{1}}=-1\right\}} \frac{p_{i_{1}}}{q_{i_{1}}} \prod_{\left\{i_{2}: z_{i_{2}}=1\right\}} \frac{q_{i_{2}}}{p_{i_{2}}}
$$

3. Set

$$
\mathbf{x}^{(t+1)}=\left\{\begin{array}{ccc}
\mathbf{x}^{\prime} & \text { with probability } & \alpha\left(\mathbf{x}^{(t)}, \epsilon\right) \\
\mathbf{x}^{(t)} & \text { with probability } & 1-\alpha\left(\mathbf{x}^{(t)}, \epsilon\right)
\end{array}\right.
$$

- End for

For the additive transformation, the issues are further simplified. The random variable $z_{i}$ here takes the value -1 and 1 with probabilities $p_{i}$ and $q_{i}=1-p_{i}$, respectively. So, only $p_{i}$ needs to be specified. Since $z_{i}=0$ has probability zero in this setup, there is no need to perform rejection sampling to reject any move-type.

\subsection{Discussion on choices of $p_{i}$ and $q_{i}$}

Interestingly, the ideas developed in Section 3.1 provide us with a handle to control the move-type probabilities, by simply controlling $p_{i}$ and $q_{i}$ for each $i$. For instance, if some pilot MCMC analysis tells us that $x_{i}$ and $x_{j}$ are highly positively correlated, then we could set $p_{i}$ and $p_{j}$ (or $q_{i}$ and $q_{j}$ ) to be high provided the forward transformation on both $x_{i}$ and $x_{j}$ are increasing. On the other hand, if $x_{i}$ and $x_{j}$ are highly negatively correlated, then we can set $p_{i}$ to be high (low) and $q_{j}$ to be low (high) and so on. Apart from these choices, there are theoretically motivated choices of $p_{i}$ and $q_{i}$ as well. Indeed, Dey and Bhattacharya (2013) prove, under suitable regularity conditions, that additive TMCMC is geometrically ergodic when $p_{i}=q_{i}=1 / 2$. Thus, at least for additive transformations, the choice $p_{i}=q_{i}=1 / 2$ for $i=1 \ldots, k$, seems to be reasonable from a theoretical perspective. In our TMCMC illustration of the Challenger data presented in Section 4 we choose $p_{i}, q_{i}$ based on the posterior correlations obtained from a pilot MCMC analysis, whereas in the case of Rongelap data we set $p_{i}=q_{i}=1 / 2$. 


\subsubsection{Dependence structure on $\mathrm{z}$}

The procedure outlined above simulates each co-ordinate $z_{i}$ independently, for $i=1, \ldots, k$. But because the same $\epsilon$ is used for the transformation of each co-ordinate $x_{i}$ of $x$, the co-ordinate moves are dependent. However in addition, it is also possible to consider dependence between the components of $\mathbf{z}$ using a hierarchical structure. For example, for $i=1, \ldots, k$, let $\mathbf{w}_{i}=\left(w_{i 1}, \ldots, w_{i k}\right) \sim$ $N\left(\boldsymbol{\mu}_{i}, \boldsymbol{\Sigma}_{i}\right) ; i=1,2,3$, where the parameters $\left(\boldsymbol{\mu}_{i}, \boldsymbol{\Sigma}_{i}\right) ; i=1,2,3$ are assumed to be known. We then set $p_{i}=\exp \left(w_{1 i}\right) / \sum_{j=1}^{3} \exp \left(w_{j i}\right), q_{i}=\exp \left(w_{2 i}\right) / \sum_{j=1}^{3} \exp \left(w_{j i}\right)$, so that $1-p_{i}-q_{i}=$ $\exp \left(w_{3 i}\right) / \sum_{j=1}^{3} \exp \left(w_{j i}\right)$. These $k$-variate normal distributions induce dependence between $\mathbf{p}=\left(p_{1}, \ldots, p_{k}\right)$ and $\mathbf{p}=\left(q_{1}, \ldots, q_{k}\right)$. Thus, even though conditionally on $\left\{\left(p_{i}, q_{i}\right) ; i=1, \ldots, k\right\} z_{i}$ are independent, marginalized over $\mathbf{p}$ and $\mathbf{q}$, the components of $\mathbf{z}$ are dependent. To achieve the effect of this dependent structure in TMCMC in a theoretically valid manner, at each iteration of the TMCMC algorithm we can simulate $\mathbf{w}_{1}, \mathbf{w}_{2}, \mathbf{w}_{3}$ from their respective $k$-variate normal distributions, and from the simulated values obtain, for $i=1, \ldots, k, p_{i}=\exp \left(w_{1 i}\right) / \sum_{j=1}^{3} \exp \left(w_{j i}\right)$, and $q_{i}=\exp \left(w_{2 i}\right) / \sum_{j=1}^{3} \exp \left(w_{j i}\right)$. To avoid getting $p_{i}$ and $q_{i}$ too close to zero in some simulations, we can appropriately truncate the $k$-variate normal distributions. Once $\left\{\left(p_{i}, q_{i}\right) ; i=1, \ldots, k\right\}$ are obtained, conditionally on these probabilities, $z_{i} ; i=1, \ldots, k$ will be simulated independently. Thus, the algorithm for dependent $\mathbf{z}$ admits the same form as Algorithm 3.1; only in the first step, simulation of $\mathbf{p}$ and $\mathbf{q}$ from their respective dependent distributions must precede independent simulation of $z_{i} ; i=1, \ldots, k$. The algorithm (Algorithm S-4.1) and proof of detailed balance are provided in Sections S-4 and S-5, respectively.

Note that for the additive transformation, since $q_{i}=1-p_{i}$, only the joint distribution of $\mathbf{p}$ needs to be considered, with $p_{i}=\exp \left(w_{1 i}\right) / \sum_{j=1}^{2} \exp \left(w_{j i}\right)$, and it is not necessary to simulate $\mathbf{w}_{3}$ at all.

Theoretically appropriate choices of $\left(\boldsymbol{\mu}_{i}, \boldsymbol{\Sigma}_{i}\right) ; i=1,2,3$ will be our topic of future research but from a practical point of view, one can tune these parameters to achieve good mixing properties and acceptance rates.

\subsection{Advantages of TMCMC updating with single $\epsilon$}

Standard methods like sequential RWMH may tend to be computationally infeasible in high dimensions while inducing mixing problems due to posterior dependence between the parameters, whereas TMCMC remains free from the aforementioned problems thanks to singleton $\epsilon$ and joint updating of all the parameters. Specialized proposals for joint updating may be constructed for specific problems only; for 
instance, block updating proposals for Gaussian Markov random fields are available (Rue (2001)). But generally, efficient block updating proposals are not available. Moreover, even in the specific problems, simulation from the specialized block proposals and calculating the resulting acceptance ratio are generally computationally very expensive. In contrast, TMCMC with singleton $\epsilon$ seems to be much more general and efficient. Moreover, we demonstrate in Section 4 in connection with the Challenger data problem that TMCMC can outperform well-established block proposal mechanisms, usually based on the asymptotic covariance matrix of the maximum likelihood estimator (MLE), in terms of acceptance rate.

\section{Application of TMCMC to the Challenger dataset}

In 1986, the space shuttle Challenger exploded during take off, killing the seven astronauts aboard. The explosion was the result of an O-ring failure, a splitting of a ring of rubber that seals the parts of the ship together. The accident was believed to be caused by the unusually cold weather $\left(31^{0} \mathrm{~F}\right.$ or $\left.0^{0} \mathrm{C}\right)$ at the time of launch, as there is reason to believe that the O-ring failure probabilities increase as temperature decreases. The data are provided in Table S-1 for ready reference. We shall analyze the data with the help of well-known logit model. Our main aim is not analyzing and drawing inference since it is done already in Dalal et al. (1989), Martz and Zimmer (1992) and Robert and Casella (2004) . We shall rather compare the different MCMC methodologies used in Bayesian inference for logit-model. Let

$$
\eta_{i}=\beta_{1}+\beta_{2} x_{i}
$$

where $x_{i}=t_{i} / \max t_{i}, t_{i}$ 's being the temperature at flight time (degrees $\left.\mathrm{F}\right), i=1, \ldots, n$. and $n=23$. Also suppose $y_{i}$ is the indicator variable denoting failure of 0 -ring. We suppose $y_{i}$ 's independently follow $\operatorname{Bernoulli}\left(\pi\left(x_{i}\right)\right)$.

In the logit model we suppose that the log-odd ratio is a linear function of temperature at flight time, i.e.,

$$
\log \frac{\pi}{1-\pi}=\eta=\beta_{1}+\beta_{2} x
$$

which gives

$$
\pi_{i}=\exp \left(\eta_{i}\right) /\left(1+\exp \left(\eta_{i}\right)\right)
$$

In the absence of information regarding $\left(\beta_{1}, \beta_{2}\right)$, we specify a uniform (improper) prior for $\left(\beta_{1}, \beta_{2}\right)$. 


\begin{tabular}{|c|c|c|c|c|c|c|c|c|c|}
\hline variable & method & $\begin{array}{c}\text { acceptance rate } \\
(\%)\end{array}$ & mean & std & $2.5 \% *$ & $25 \% *$ & $50 \% *$ & $75 \% *$ & $97.5 \% *$ \\
\hline \multirow{3}{*}{$\beta_{1}$} & RWMH & 42.17 & 19.119 & 8.078 & 4.909 & 13.481 & 18.475 & 24.227 & 38.176 \\
\hline & MH & 42.60 & 18.930 & 8.513 & 5.011 & 12.823 & 17.981 & 23.957 & 38.206 \\
\hline & TMCMC & 73.23 & 18.973 & 7.944 & 4.970 & 12.881 & 16.210 & 21.685 & 37.877 \\
\hline \multirow{3}{*}{$\beta_{2}$} & RWMH & 48.14 & -23.724 & 9.613 & -46.272 & -29.786 & -22.984 & -17.019 & -6.7792 \\
\hline & MH & 42.60 ** & -23.491 & 10.128 & -46.461 & -29.464 & -22.353 & -16.261 & -6.956 \\
\hline & TMCMC & $73.23 * *$ & -23.165 & 9.762 & -46.404 & -28.891 & -22.282 & -16.446 & -7.026 \\
\hline
\end{tabular}

Table 4.1: Summary of the posterior samples based on MCMC runs of length 100,000 out of which first 20,000 samples are discarded as burn-in.

RWMH = Random walk Metropolis-Hastings, $\mathrm{MH}=$ Metropolis-Hastings with bivariate normal proposal, TM$\mathrm{CMC}=\mathrm{MCMC}$ based on transformation

$*$ : posterior sample quantiles.

**: same as acceptance ratio for $\beta_{1}$ since updated jointly.

We construct an appropriate additive transformation $T: \mathbb{R}^{2} \times \mathbb{R} \rightarrow \mathbb{R}^{2}$ as follows. First, we consider the form $T_{(1,1)}\left(\left(\beta_{1}, \beta_{2}\right), \epsilon\right)=\left(\beta_{1}, \beta_{2}\right)^{\prime}+\left(s_{1} \epsilon, s_{2} \epsilon\right)^{\prime}$, where $s_{1}$ and $s_{2}$ are the standard errors of the maximum likelihood estimator of $\left(\beta_{1}, \beta_{2}\right)^{\prime}$. Thus, we finally obtain the transformation

$$
T_{(1,1)}\left(\left(\beta_{1}, \beta_{2}\right), \epsilon\right)=\left(\beta_{1}+7.3773 \epsilon, \beta_{2}+4.3227 \epsilon\right)
$$

and use Algorithm 3.1 with $\mathcal{Y}=(0, \infty)$ and

$$
g(\epsilon) \propto \exp \left(-\epsilon^{2} / 2\right), \epsilon>0
$$

that is, the $N(0,1)$ distribution truncated to the left at zero. From the covariance matrix $\mathbf{C}$ we observe that the correlation of $\hat{\beta}_{1}$ and $\hat{\beta}_{2}$ is approximately -0.99 and hence from our discussion in Section 3.2, setting high probabilities to the moves $T_{(1,-1)}(\mathbf{x}, \epsilon)$ and $T_{(-1,1)}(\mathbf{x}, \epsilon)$ should facilitate good mixing. Following the discussion in Section 3.2 we set $P((1,1))=P((-1,-1))=0.01$ and $P((1,-1))=P((-1,1))=0.49$.

Also for comparison we use the RWMH algorithm (both joint and sequential updation) and also the MH algorithm with proposal $q\left(\boldsymbol{\beta}^{\prime} \mid \boldsymbol{\beta}\right)=N(\boldsymbol{\beta}, \boldsymbol{\Sigma})$ where $\boldsymbol{\Sigma}=h^{2} \mathbf{C}$ (we take $h=1$ for our purpose) with $\mathrm{C}$ being the large sample covariance matrix of the MLE $\widehat{\beta}$ of $\beta$. Table 4.1 gives the posterior summaries and Figure 4.1 gives the trace plots of $\beta_{1}$ and $\beta_{2}$ for TMCMC sampler and the MH sampler. It is seen that the mixing is excellent even though a single $\epsilon$ has been used.

Notice the excellent result of the MCMC based on transformations. The acceptance ratio is almost twice as large as those for other two MH algorithms. As remarked in Section 3.3, indeed TMCMC 

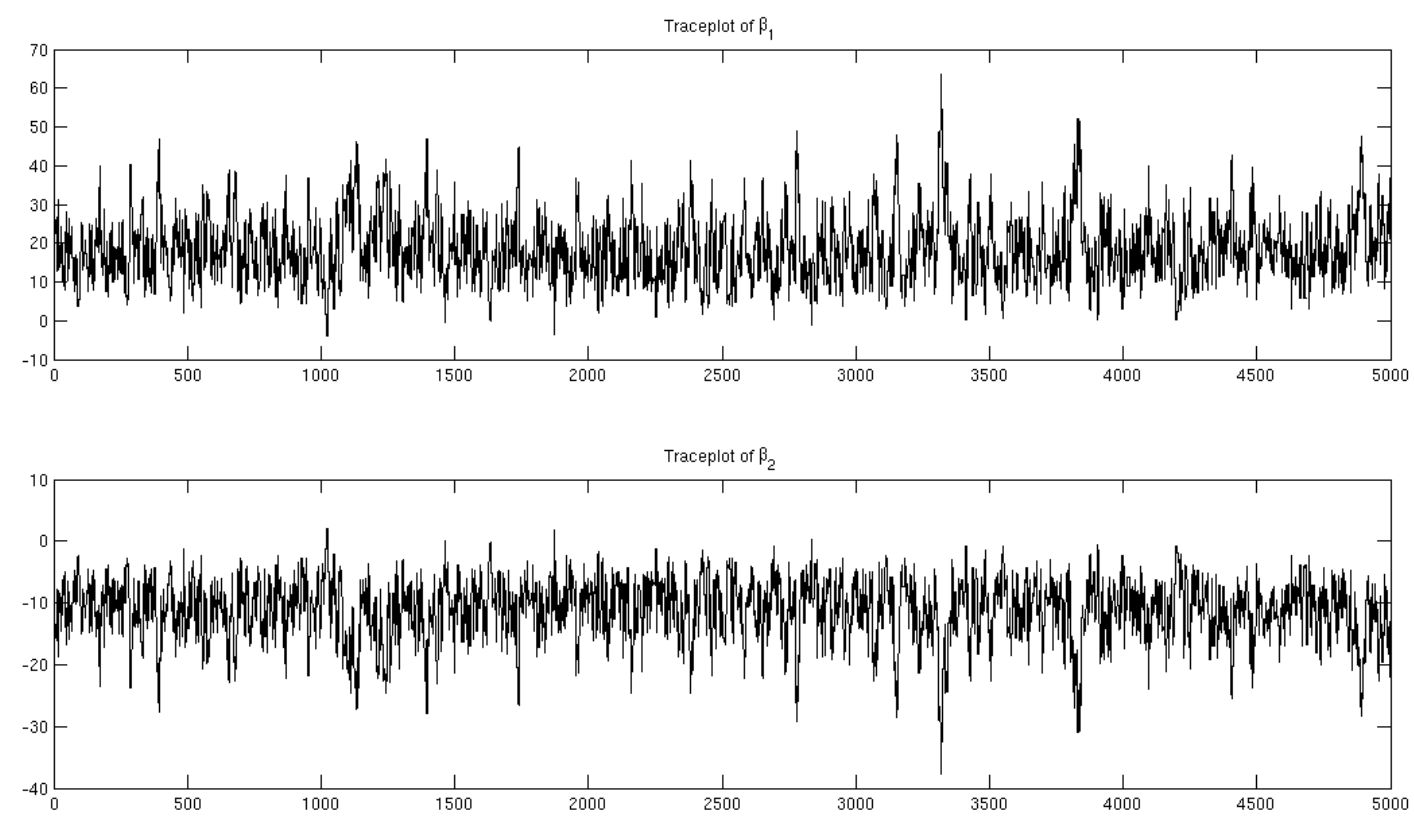

(a)
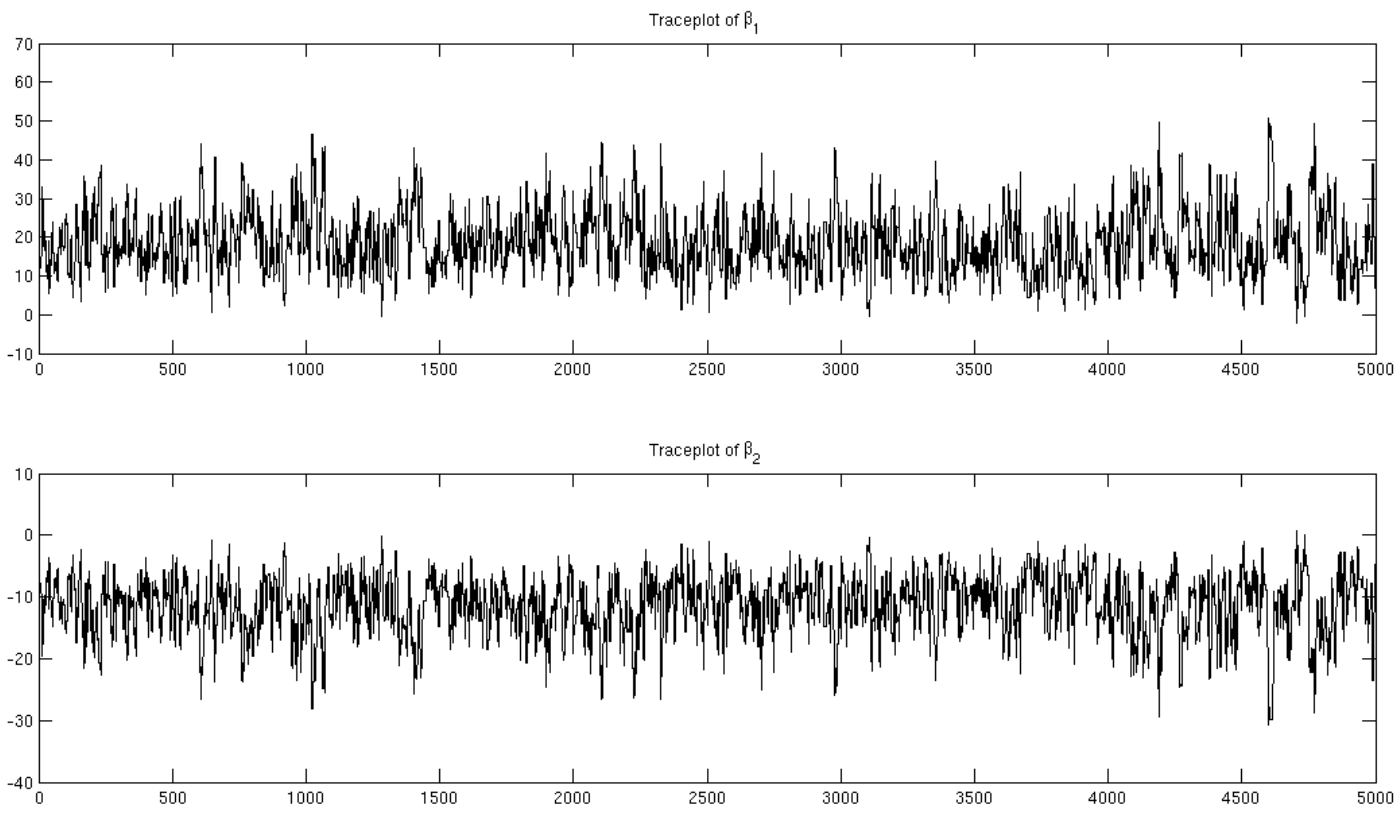

(b)

Figure 4.1: Trace plots of $\beta_{1}$ and $\beta_{2}$ (a) TMCMC (b) MH 
outperformed the MH block proposal based on the large sample covariance matrix of the MLE of $\beta$ in terms of acceptance rate. Also for implementing TMCMC we need to simulate only one $\epsilon$ in each step. In the RWMH with sequential updating and in $\mathrm{MH}$ based on bivariate normal proposal we need two such $\epsilon$ 's. In the RWMH we need to calculate the likelihood twice in each iteration. So, TMCMC dominates

the other two in this respect. It can be easily anticipated, in light of the theoretical arguments regarding acceptance rate presented in Section S-6, that for joint RWMH the acceptance rate would be even lower. In Section 5, where we consider a 160-dimensional problem, we show that, that TMCMC outperforms joint RWMH by a substantially large margin in terms of acceptance rate.

\section{Application of TMCMC to the geostatistical problem of radionu- clide concentrations on Rongelap Island}

\subsection{Model and prior description}

We now consider the much analyzed radionuclide count data on Rongelap Island (see, for example, Diggle et al. (1997), Diggle et al. (1998), Christensen (2004), Christensen (2006)), and illustrate the performance of TMCMC with a singleton $\epsilon$. For $i=1, \ldots, 157$, Diggle et al. (1998) model the count data as

$$
Y_{i} \sim \operatorname{Poisson}\left(M_{i}\right)
$$

where

$$
M_{i}=t_{i} \exp \left\{\beta+S\left(\mathbf{x}_{i}\right)\right\}
$$

$t_{i}$ is the duration of observation at location $\mathbf{x}_{i}, \beta$ is an unknown parameter and $S(\cdot)$ is a zero-mean Gaussian process with isotropic covariance function of the form

$$
\operatorname{Cov}\left(S\left(\mathbf{x}_{1}^{\star}\right), S\left(\mathbf{x}_{2}^{\star}\right)\right)=\sigma^{2} \exp \left\{-\left(\alpha\left\|\mathbf{x}_{1}^{\star}-\mathbf{x}_{2}^{\star}\right\|\right)^{\delta}\right\}
$$

for any two locations $\mathbf{x}_{1}^{\star}, \mathbf{x}_{2}^{\star}$. In the above, $\|\cdot\|$ denotes the Euclidean distance between two locations, and $\left(\sigma^{2}, \alpha, \delta\right)$ are unknown parameters. Typically in the literature $\delta$ is set equal to 1 (see, $e$. $g$. Christensen (2006)), which we adopt. We assume uniform priors on the entire parameter space corresponding to $\left(\beta, \log \left(\sigma^{2}\right), \log (\alpha)\right)$. 
We remark that since the Gaussian process $S(\cdot)$ does not define a Markov random field, the block updating proposal developed by Rue (2001) is not directly applicable here. Rue (2009) attempt to develop deterministic approximations to latent Gaussian models, but the scope of such approximations is considerably restricted by the conditional independence (Gaussian Markov random field) assumption (Banerjee (2009)). Thanks to the generality and efficiency of our proposed methodology, it seems most appropriate to fit the Rongelap island model using TMCMC with singleton $\epsilon$.

\subsection{Results of additive TMCMC with singleton $\epsilon$}

Drawing $\epsilon \sim N(0,1) \mathbb{I}(\epsilon>0)$, we considered the following additive transformation

$$
\begin{aligned}
T(\beta, \epsilon) & =\beta \pm 2 \epsilon \\
T\left(\log \left(\sigma^{2}\right), \epsilon\right) & =\log \left(\sigma^{2}\right) \pm 5 \epsilon \\
T(\log (\alpha), \epsilon) & =\log (\alpha) \pm 5 \epsilon \\
T\left(S\left(\mathbf{x}_{i}\right), \epsilon\right) & =S\left(\mathbf{x}_{i}\right) \pm 2 \epsilon ; \text { for } i=1, \ldots, 157
\end{aligned}
$$

The scaling factors associated with $\epsilon$ in each of the transformations are chosen on a trial-and-error basis after experimenting with several initial (pilot) runs of TMCMC. We assigned equal probabilities to all the $2^{160}$ move types. Move types are selected by independently generating $z_{i}$ taking the values +1 and -1 with equal probabilities, that is, we set $p_{i}=q_{i}=1 / 2$ for $i=1, \ldots, 160$. As mentioned in Section 3.2 this choice of equal probabilities of forward and backward transformation is motivated by our result on geometric ergodicity.

After discarding the first $2 \times 10^{7}$ iterations as burn-in, we stored 1 in every 100 iterations in the next $3.5 \times 10^{7}$ iterations. This entire simulation took about a week to run on an ordinary laptop machine and about 3 days on a workstation. The autocorrelation functions of the variables (after further thinning by 10) of our TMCMC run, displayed in Figure 5.1, indicates reasonable mixing properties. The acceptance rate, after discarding the burn-in period, is $0.43 \%$ (considering the complete run of TMCMC after burnin, that is, including thinning as well).

\subsection{Comparison with joint RWMH}

We also implemented a joint RWMH using the same additive transformation as in Section 5.2 but with different $\epsilon$ 's for each unknown. Now the acceptance rate reduced to $0.0005 \%$. These observations are 


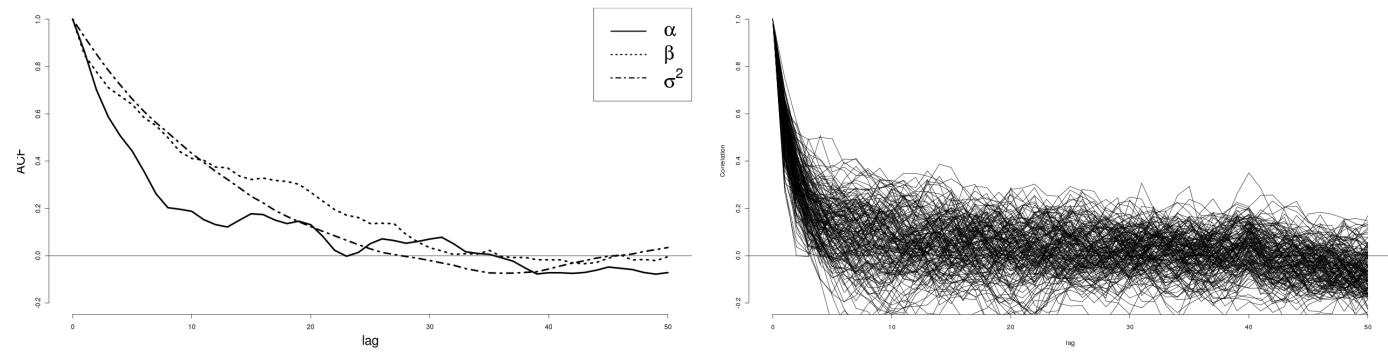

Figure 5.1: Autocorrelation plots of the variables, $\alpha, \beta, \log \sigma^{2}$ (left-panel) and $s_{1}, \ldots, s_{157}$ (right-panel) in the TMCMC run.

broadly in keeping with the theoretical discussions presented in Section S-6.

\section{Application of TMCMC to doubly-intractable problem}

Doubly-intractable distributions arise quite frequently in fields like circular statistics, directed graphical models, Markov point processes etc. Even some standard distributions like gamma and beta involve intractable normalizing constants. Formally, a density $h(\mathbf{y} \mid \theta)$ of the data set $\mathbf{y}=\left(y_{1}, \ldots, y_{n}\right)^{\prime}$ is said to be doubly-intractable if it is of the form

$$
h(\mathbf{y} \mid \theta)=f(\mathbf{y} \mid \theta) / Z(\theta)
$$

where $Z(\theta)$ is a function that is not available in closed form. So if we put a prior $\pi(\theta)$ on $\theta$, then the posterior is given by

$$
\pi(\theta \mid \mathbf{y})=\frac{1}{c(\mathbf{y})} \frac{f(\mathbf{y} \mid \theta)}{Z(\theta)} \pi(\theta) \quad \text { where } \quad c(\mathbf{y})=\int_{\Theta} \frac{f(\mathbf{y} \mid \theta)}{Z(\theta)} \pi(\theta) d \theta
$$

Thus, if we try to apply MH like algorithms then the acceptance ratio will involve ratio of the function $Z(\cdot)$ at two parameter points $\theta$ and $\theta^{\prime}$. Hence directly applying $\mathrm{MH}$ may not be feasible. Works by Møller et al. (2004) and Murray et al. (2006) are significant in this field. A double MH sampler approach is taken in Liang (2010). In this section we briefly discuss the bridge-exchange algorithm by Murray et al. (2006) and show how our application of TMCMC in the bridge-exchange algorithm may facilitate fast computation.

Suppose $M \in \mathbb{N}$ is the bridge size, $\beta_{m}=m /(M+1), m=0, \ldots, M$. Define the density

$$
p_{m}\left(\mathbf{x} \mid \theta, \theta^{\prime}\right) \propto f(\mathbf{x} \mid \theta)^{\beta_{m}} f\left(\mathbf{x} \mid \theta^{\prime}\right)^{1-\beta_{m}} \equiv f_{m}\left(\mathbf{x} \mid \theta, \theta^{\prime}\right), \quad m=0, \ldots, M
$$


Obviously, $\mathbf{x}$ is of the same dimensionality as $\mathbf{y}$; that is, $\mathbf{x}=\left(x_{1}, \ldots, x_{n}\right)^{\prime}$. Further suppose that for each $m, T_{m}\left(\mathbf{x} \rightarrow \mathbf{x}^{\prime} \mid \theta, \theta^{\prime}\right)$ is a kernel satisfying the detailed balance condition

$$
T_{m}\left(\mathbf{x} \rightarrow \mathbf{x}^{\prime} \mid \theta, \theta^{\prime}\right) p_{m}\left(\mathbf{x} \mid \theta, \theta^{\prime}\right)=T_{m}\left(\mathbf{x}^{\prime} \rightarrow \mathbf{x} \mid \theta, \theta^{\prime}\right) p_{m}\left(\mathbf{x}^{\prime} \mid \theta, \theta^{\prime}\right)
$$

Now with a proposal density $q\left(\theta \rightarrow \theta^{\prime} \mid \mathbf{y}\right)$ for the parameter, the bridge-exchange algorithm is given below.

Algorithm 6.1 The bridge-exchange algorithm

- Input: initial state $\theta_{0}$, length of the chain $N$, \#bridge levels $M$.

- For $t=0, \ldots, N-1$

1. Propose $\theta^{\prime} \sim q\left(\theta^{\prime} \leftarrow \theta_{t} \mid \mathbf{y}\right)$

2. Generate an auxiliary variable with exact sampling:

$$
\mathbf{x}_{0} \sim p_{0}\left(\mathbf{x}_{0} \mid \theta, \theta^{\prime}\right) \equiv f\left(\mathbf{x}_{0} \mid \theta^{\prime}\right) / Z(\theta)
$$

3. Generate M further auxiliary variables with transition operators:

$$
\begin{aligned}
\mathbf{x}_{1} & \sim T_{1}\left(\mathbf{x}_{0} \rightarrow \mathbf{x}_{1} \mid \theta, \theta^{\prime}\right) \\
\mathbf{x}_{2} & \sim T_{2}\left(\mathbf{x}_{1} \rightarrow \mathbf{x}_{2} \mid \theta, \theta^{\prime}\right) \\
& \vdots \\
\mathbf{x}_{M} & \sim T_{M}\left(\mathbf{x}_{M-1} \rightarrow \mathbf{x}_{M} \mid \theta, \theta^{\prime}\right)
\end{aligned}
$$

4. Compute

$$
\alpha\left(\theta^{\prime} \leftarrow \theta_{t}\right)=\frac{q\left(\theta^{\prime} \rightarrow \theta \mid \mathbf{y}\right) \pi\left(\theta^{\prime}\right) f\left(\mathbf{y} \mid \theta^{\prime}\right)}{q\left(\theta \rightarrow \theta^{\prime} \mid \mathbf{y}\right) \pi(\theta) f(\mathbf{y} \mid \theta)} \prod_{m=0}^{M} \frac{f_{m+1}\left(\mathbf{x}_{m} \mid \theta, \theta^{\prime}\right)}{f_{m}\left(\mathbf{x}_{m} \mid \theta, \theta^{\prime}\right)}
$$

5. Set

$$
\theta_{t+1}=\left\{\begin{array}{ccc}
\theta^{\prime} & \text { with probability } & \alpha\left(\theta^{\prime} \leftarrow \theta_{t}\right) \\
\theta_{t} & \text { with probability } & 1-\alpha\left(\theta^{\prime} \leftarrow t_{t}\right)
\end{array}\right.
$$


Now we see that, since each of the auxiliary variables $\mathbf{x}_{m}, m=1, \ldots, M$, is $n$-dimensional, generation of these auxiliary variables may be computationally demanding if the sample size $n$ is moderate or large especially when one has to simulate from the sample space using accept-reject algorithms as in the case of circular variables. For any kernel $T_{m}$ which is not based on TMCMC, $O(n M)$ variables are required to be generated from the state-space per iteration. Appealing to TMCMC, recall that with the additive transformation with a single $\epsilon$, the kernel still satisfies the detailed balance condition.

We assume that $\mathcal{X}$ is a group under some binary operation and that there is a homomorphism from $\left(\mathbb{R}^{p},+\right)$ to $\mathcal{X}$ for some $p \in \mathbb{N}$. So we denote the binary operation on $\mathcal{X}$ by ' + ' itself. Let $g$ be a density on $\mathcal{X}$. We construct the kernels $T_{m}$ as follows:

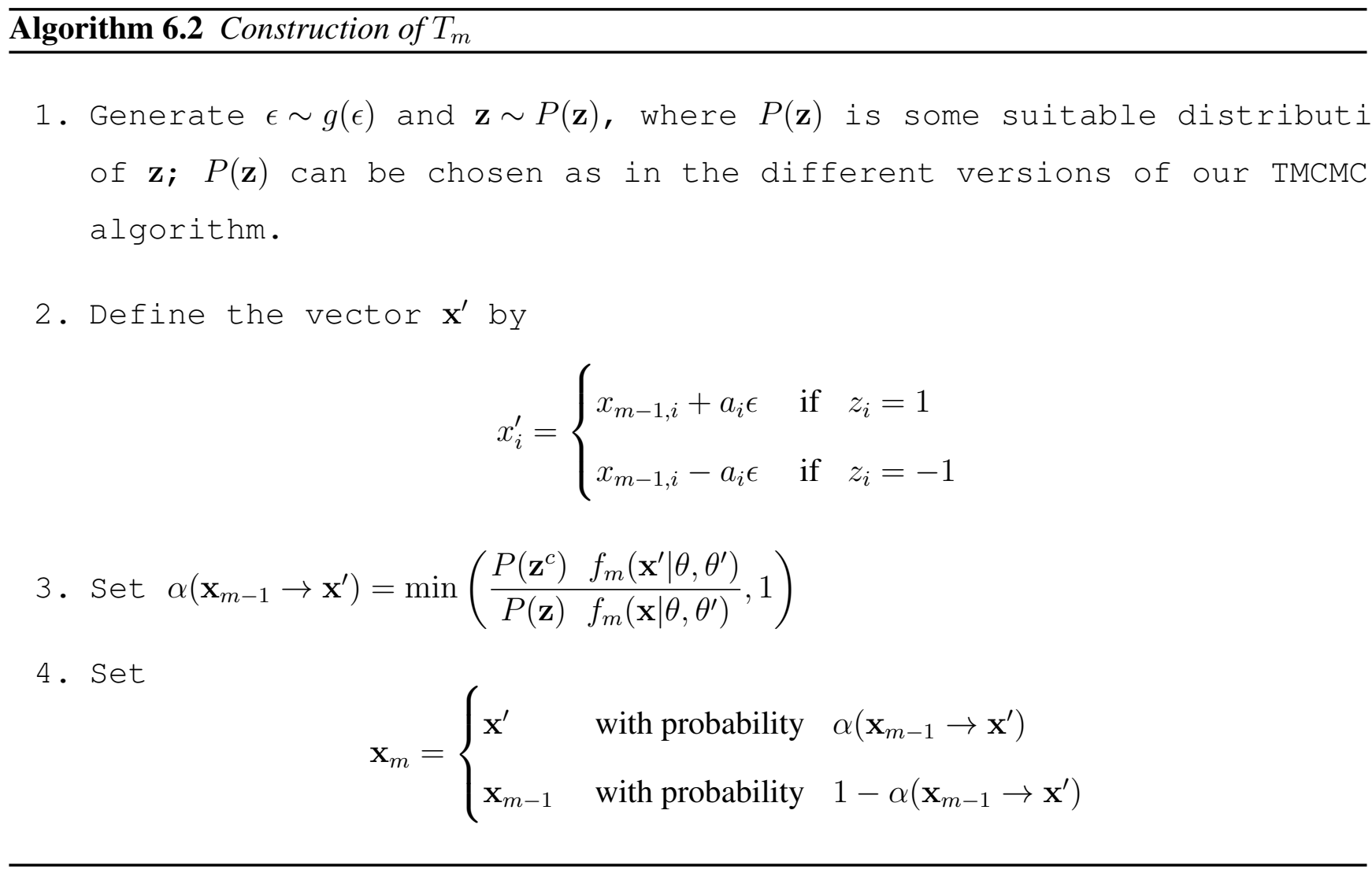

In this way we need only $O(M)$ simulations per iteration. Homomorphism from $\left(\mathbb{R}^{p},+\right)$ to $\mathcal{X}$ holds in many cases, for example, in circular models where the state-space is $(-\pi, \pi]$ is a group with respect to addition modulo $\pi$. 

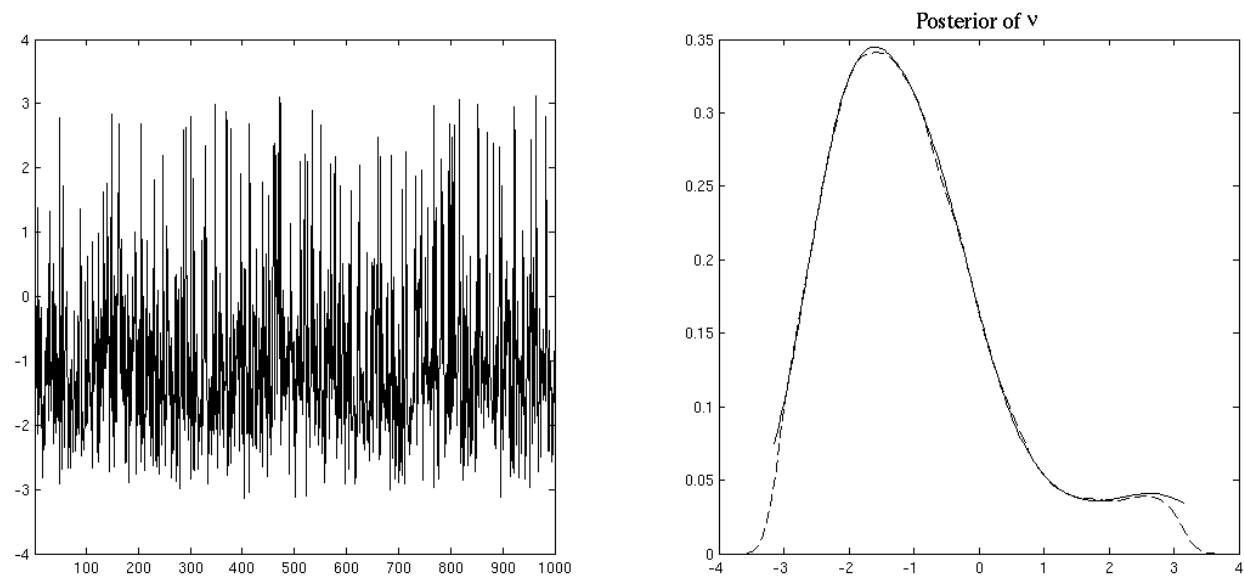

Figure 6.1: Left panel: Trace plot of last 1,000 samples. Right panel: exact posterior density of $\nu$ (solid line) and it's estimate (dash-dotted line).

\subsection{Simulation study to illustrate TMCMC in bridge-exchange algorithm}

Here we illustrate our method for a circular model of the form

$$
h(y \mid \nu)=\frac{1}{Z(\nu)} \exp (\cos (y+\nu \sin (y))),-\pi<y, \nu \leq \pi
$$

We generate a sample of size 20 from $h(y \mid \nu=0)$ and estimate the parameter $\nu$ based on this sample. The prior chosen on $\nu$ is the uniform distribution on $(-\pi, \pi]$ and $g(\cdot)$ is chosen to be the normal distribution with mean 0 and variance 1 restricted on the set $(0, \pi]$. Since the components of $\mathbf{x}_{0}$ are $i i d$, we used $\operatorname{Pr}\left(Z_{i}=1\right)=\operatorname{Pr}\left(Z_{i}=-1\right)=1 / 2$ and $a_{i}=1$ for each $i$. We set $M=100$ and chose $q\left(\nu^{\prime} \mid \nu\right)$ to be the Von-mises distribution with mean $\nu$ and concentration 0.5 to keep the acceptance level around $63 \%$.

The right panel of Figure 6.1 shows that the estimated posterior density of $\nu$ is very close to the exact posterior density. The little discrepancy at the tails are due to the fact that $\nu$ is a circular variable and hence its support is $(-\pi, \pi]$ and the density is not zero at the end points - a fact that is not incorporated in the kernel density estimator. The left panel of the same figure shows that the mixing is excellent. Notice that here we have saved $100(n M-M) / n M=95 \%$ simulations. 


\section{Summary, conclusions and future work}

In this paper we have proposed a novel MCMC method that uses deterministic transformations and move types to update the Markov chain. We have shown that our algorithm TMCMC generalizes the MH algorithm boiling down to $\mathrm{MH}$ with a specialized proposal density in one-dimensional cases. For higher dimensions if each component $x_{i}$ of the random vector to be updated is associated with a distinct $\epsilon_{i}$, then TMCMC again boils down to the MH algorithm with a specialized proposal density. But in dimensions greater than one, with less number of distinct $\epsilon_{i}$ than the size of the random vector to be updated, TMCMC does not admit any MH representation. Several versions of TMCMC have been detailed in this paper and in the supplement. In Section S-6 of the supplement, under reasonable regularity conditions we have provided and compared the asymptotic forms of the acceptance rates of RWMH and additive TMCMC when the dimensionality increases to infinity and have shown that the latter converges to zero at a much slower rate. That HMC is also a special case of TMCMC, is also explained in Section S-7; in addition, we have provided asymptotic forms of the acceptance rate of HMC under reasonable regularity conditions and have shown that, as the dimensionality grows to infinity, the forms converge to zero at much faster rates than additive TMCMC. In Section S-8 we also contrasted TMCMC with the transformation-based methods of Liu and Yu (1999), Liu and Sabatti (2000), and Kou et al. (2005).

The advantages of TMCMC are more prominent in high dimensions, where simulating a single random variable can update many parameters at the same time, thus saving a lot of computing resources. That many variables can be updated in a single block without compromising much on the acceptance rate, seems to be another quite substantial advantage provided by our algorithm. We illustrated with examples that TMCMC can outperform MH significantly, particularly in high dimensions. The computational gain of using TMCMC for simulations from doubly intractable distributions, is also significant, and is illustrated with an example.

In this article we have developed TMCMC for continuous state spaces. However, in Section S-9 we show how TMCMC can be generalized to discrete state spaces as well. A complete development of TMCMC for discrete cases will be a subject of our future work. 


\section{Acknowledgment}

We sincerely thank the reviewer whose comments have led to an improved version of our manuscript. Conversations with Dr. Ranjan Maitra has also led to improved presentation of some of the ideas.

\section{References}

S. Banerjee. Discussion: Approximate Bayesian Inference for Latent Gaussian Models by using Integrated Nested Laplace Approximations. Journal of the Royal Statistical Society. Series B, 71:365, 2009.

O. F. Christensen. Monte Carlo Maximum Likelihood in Model-Based Geostatistics. Journal of Computational and Graphical Statistics, 13:702-718, 2004.

O. F. Christensen. Robust Markov Chain Monte Carlo Methods for Spatial Generalized Linear Mixed Models. Journal of Computational and Graphical Statistics, 15:1-17, 2006.

S. R. Dalal, E. B. Fowlkes, and B. Hoadley. Risk Analysis of the Space Shuttle: pre-Challenger Prediction of Failure. Journal of the American Statistical Association, 84:945-957, 1989.

K. K. Dey and S. Bhattacharya. On Geometric Ergodicity of Additive Transformation Based Markov Chain Monte Carlo. Technical report, Indian Statistical Institute, 2013.

P. J. Diggle, J. A. Tawn, and R. A. Moyeed. Geostatistical Analysis of Residual Contamination from Nuclear Weapons Testing. In V. Barnet and K. F. Turkman, editors, Statistics for Environment 3: Pollution Assessment and Control, pages 89-107. Chichester: Wiley, 1997.

P. J. Diggle, J. A. Tawn, and R. A. Moyeed. Model-Based Geostatistics (with discussion). Applied Statistics, 47:299-350, 1998.

S. Dutta. Multiplicative Random Walk Metropolis-Hastings on the Real Line. Sankhya B, 74:315-342, 2012.

S. Dutta and S. Bhattacharya. Supplement to "Markov Chain Monte Carlo Based on Deterministic Transformations", 2013. Submitted. 
Y. Guan and S. M. Krone. Small-World MCMC and Convergence to Multi-Modal Distributions: From Slow Mixing to Fast Mixing. The Annals of Applied Probability, 17:284-304, 2007.

S. C. Kou, X. S. Xie, and J. S. Liu. Bayesian Analysis of Single-Molecule Experimental Data. Applied Statistics, 54:469-506, 2005.

F. Liang. A Double Metropolis-Hastings Sampler for Spatial Models with Intractable Normalizing Constants. Journal of Statistical Computation and Simulation, 80:1007-1022, 2010.

J. Liu. Monte Carlo Strategies in Scientific Computing. Springer-Verlag, New York, 2001.

J. S. Liu and S. Sabatti. Generalized Gibbs Sampler and Multigrid Monte Carlo for Bayesian Computation. Biometrika, 87:353-369, 2000.

J. S. Liu and Y. N. Yu. Parameter Expansion for Data Augmentation. Journal of the American Statistical Association, 94:1264-1274, 1999.

H. F. Martz and W. J. Zimmer. The Risk of Catastrophic Failure of the Solid Rocket Boosters on the Space Shuttle. The American Statistician, 46:42-47, 1992.

J. Møller, A. N. Pettitt, K. K. Berthelsen, and R. W. Reeves. An Efficient Markov Chain Monte Carlo Method for Distributions with Intractable Normalising Constants. Technical report, Department of Mathematical Sciences, Aalborg University, 2004.

I. Murray, Z. Ghahramani, and D. J. C MacKay. MCMC for Doubly-Intractable Distributions. In R. Dechter and T. S. RIchardson, editors, Proceedings of the 22nd Annual Conference on Uncertainty in Artificial Intelligence (UAI-06), pages 359-366. AUAI Press, 2006.

C. P. Robert and G. Casella. Monte Carlo Statistical Methods. Springer-Verlag, New York, 2004.

H. Rue. Fast sampling of Gaussian Markov random fields. Journal of the Royal Statistical Society. Series $B, 63: 325-338,2001$.

H. Rue. Approximate Bayesian Inference for Latent Gaussian Models by Using Integrated Nested Laplace Approximations. Journal of the Royal Statistical Society. Series B, 71:319-392, 2009. 